Pasquale Santangeli ORCID iD: 0000-0002-0023-9666

Jackson Liang ORCID iD: 0000-0001-8608-8899

\title{
HOW TO PERFORM LEFT ATRIAL TRANSSEPTAL ACCESS AND CATHETER ABLATION OF ATRIAL FIBRILLATION FROM A SUPERIOR APPROACH
}

Running title: Superior Approach for AF Ablation

\author{
Pasquale Santangeli, ${ }^{1} \mathrm{MD}, \mathrm{PhD}$; Santhisri Kodali, ${ }^{1} \mathrm{MD}$; Jackson J. Liang, ${ }^{2}$ DO \\ ${ }^{1}$ Cardiac Electrophysiology Section, Cardiovascular Division, Hospital of the University of \\ Pennsylvania, Philadelphia, PA, USA \\ ${ }^{2}$ Division of Cardiology, Electrophysiology Section, University of Michigan, Ann Arbor, MI
}

Word Count: 3957.

Funding: None.

Conflicts of Interest: Dr. Santangeli is a consultant for Baylis Medical, Biosense Webster, and Abbott Medical.

Acknowledgments: The authors wish to thank Linda Bruno BEXSc, Jacqueline Hering RN, Leonard Capone RN, Kristine Conn BSN, RN, and Mitiku Tolassa CBET, BS for their expertise and support in the EP lab.

\section{Corresponding author}

Pasquale Santangeli, MD, PhD

Hospital of the University of Pennsylvania

This is the author manuscript accepted for publication and undergone full peer review but has not been through the copyediting, typesetting, pagination and proofreading process, which may lead to differences between this version and the Version of Record. Please cite this article as doi: 10.1111/jce.14294.

This article is protected by copyright. All rights reserved. 
9 Founders Pavilion - Cardiology

3400 Spruce St. Philadelphia, PA, 19104

Tel: 215-662-6005; Fax: 215-662-2879

E-mail: pasquale.santangeli@pennmedicine.upenn.edu

Key Words: atrial fibrillation; catheter ablation; interrupted inferior vena cava; transseptal access Abstract

The standard technique for percutaneous catheter ablation of atrial fibrillation (AF) involves obtaining left atrial access and catheter manipulation from an inferior transfemoral venous access. However, in patients with inferior vena cava interruption (IVC-I), a standard transfemoral venous approach is not possible. In these cases, a percutaneous approach from a superior central vein, such as the internal jugular vein or the axillary/subclavian vein can be considered. In this article, we describe the details of our technique to obtain left atrial catheterization and perform catheter ablation of AF from a superior approach. Our technique involves the use of steerable sheaths, dedicated radiofrequency (RF) wires, and intracardiac echocardiography (ICE) guidance.

\section{Introduction}

Percutaneous catheter ablation has become an established therapy to control recurrent atrial fibrillation (AF), and represents one of the most common invasive procedures performed in the electrophysiology laboratory. ${ }^{1}$ Obtaining left atrial (LA) access is requisite for any AF ablation procedure, and is a fundamental skill for the interventional electrophysiologist. $^{2}$

Access to the left heart via atrial transseptal catheterization was first described in 1959 by Ross and colleagues as an alternative technique to the conventional transaortic approach to obtain left-sided hemodynamic measurements. ${ }^{3,4}$ Subsequent technical refinements pioneered by Braunwald, ${ }^{5}$ Brockenbrough, ${ }^{6}$ and Mullins, ${ }^{7}$ and significant technological advances have further improved the ease and safety of the procedure. ${ }^{8-11}$ Nevertheless, the general technique for transseptal access has remained relatively unchanged over the years, and it involves access from an inferior transfemoral venous approach. Only until very recently, all of the available tools for transseptal catheterization, including long fixed-curve or deflectable sheaths, dilators, and

This article is protected by copyright. All rights reserved. 
needles/wires have been developed with the specific purpose of facilitating transseptal catheterization from the standard inferior transfemoral venous approach. In a small subgroup of patients such as those with congenital or acquired inferior vena cava interruption (IVC-I), ${ }^{12,13}$ LA transseptal catheterization from an inferior approach is not feasible. In these patients, transseptal access from a superior venous approach for LA mapping and ablation can be considered. We herein describe in detail our approach to LA transseptal catheterization for LA mapping and catheter ablation of AF via a superior approach in patients with IVC-I.

\section{How to Obtain Transseptal Catheterization from a Superior Approach Venous Access}

Proper selection of the superior venous access is important, as it substantially impacts the ability to obtain stable contact with the interatrial septum and the facility to advance the transseptal sheath into the LA and maintain access during mapping and ablation. In general, two venous approaches are used: the right internal jugular vein or the left axillary/subclavian vein. ${ }^{14}$ Access is typically performed under ultrasound guidance and every effort is made to puncture the vein with a very shallow angle (i.e., 20-30 degrees relative to the long axis of the vein) as this greatly improves sheath and catheter manipulation compared to a more perpendicular angle of access. The use of ultrasound guidance is also important to minimize the risk of vascular access complications, particularly in fully anticoagulated patients.

A left axillary/subclavian vein access is usually preferable to improve ergonomics and minimize fluoroscopy exposure to the operator (Figure 1). However, in patients with significant right atrial (RA) dilatation, achieving stable contact with the interatrial septum from the left axillary/subclavian route is more challenging since the body of the transseptal sheath tends to drift toward the postero-lateral aspect of the RA, which can be distant from the plane of the interatrial septum. Although this limitation can be partially overcome by the use of deflectable sheaths with larger curves, maintaining stable LA access from a left axillary/subclavian vein approach in patients with an enlarged RA remains difficult. In such cases, it is advisable to use a right internal jugular vein approach to increase proximity of the transseptal sheath body with the interatrial septum (Figure 1).

This article is protected by copyright. All rights reserved. 
Obtaining LA catheterization from other superior venous accesses, such as the right axillary/subclavian vein or the left internal jugular vein, although theoretically possible, is more difficult due to a more acute angle of access to the superior vena cava. Therefore, we have thus far employed the right axillary/subclavian vein or the left internal jugular vein only occasionally for placement of intracardiac echocardiography (ICE) or other diagnostic catheters (e.g., coronary sinus catheter).

The specific anatomical relationships relevant to the transseptal access are similar to what typically encountered with a standard left atrial transseptal catheterization from a femoral venous approach (Figure 2, see below). One important aspect that should be emphasized is that, when using a superior approach, the operators should be particularly careful to obtain proper venous access as the consequences of inadvertent puncture of the adjacent arterial vessels (carotid artery or axillary/subclavian artery) can be more serious in fully anticoagulated patients. In this regard, we believe that use of ultrasound guidance for access is crucial.

\section{Intracardiac Echocardiography}

Direct intraprocedural imaging with ICE is an essential aspect of our technique for AF ablation from a superior approach, with a pivotal role in the selection of the ideal transseptal access site, validation of access, and to allow continuous monitoring of the sheath/catheter position and early recognition of complications. When introduced from a right axillary/subclavian vein or left internal jugular vein access, a long introducer sheath is favored to overcome the acute angle of access to the superior vena cava. It is important to emphasize that when a superior approach is undertaken, catheter manipulation will be reversed compared to an inferior venous access. Therefore, once the ICE transducer is positioned in the "home view" facing the tricuspid annulus, counterclockwise (instead of clockwise) rotation applied to the catheter will bring into view sequentially: the anterior LA; the mitral valve and left atrial appendage (LAA); the left pulmonary veins (PVs); the posterior LA wall; and the right PVs.

One of the principle advantages of ICE-assisted transseptal access is the ability to determine the optimal site of puncture along the interatrial septum. In this regard, while the posterior-inferior portion of the fossa ovalis with far-field visualization of the left PVs is typically

This article is protected by copyright. All rights reserved. 
considered the ideal site of access for $\mathrm{AF}$ ablation procedures from a standard inferior venous approach, the use of this access point from a superior approach renders mapping and ablation of the septal segments of the right PVs (particularly of the right inferior PV) more challenging. In our experience, obtaining access through the more superior (i.e., superior half) and anterior portion of the fossa ovalis, defined by ICE as the plane of the fossa ovalis with far-field visualization of the LAA (Figure 3 and Video 1), significantly improves mapping and ablation of the septal segments of the right PVs with minimal risk of losing transseptal access. Once access is accomplished, ICE is valuable to define the position of the mapping/ablation catheter and its relationship with the sheath, minimizing the need for fluoroscopy, and to screen for potential periprocedural complications. Transesophageal echo (TEE) is a potential alternative to ICE to guide transseptal access from a superior approach. However, the use of TEE, although possible, may be cumbersome as it would require a separate operator and echocardiography machine at the head of the operating table.

\section{Transseptal Access Technique}

Although there are reports of successful transseptal catheterization from a superior approach using fixed-curve sheaths and a manually bent Brockenbrough transseptal needle, ${ }^{13}$ this technique is highly dependent on the individual patient anatomy and ability to engage the fossa ovalis with non-steerable sheaths and standard transseptal needles. Furthermore, advancement of the transseptal dilator and sheath into the LA over a standard needle may be difficult. Our approach to transseptal catheterization from a superior access relies on the use of steerable sheaths $\left(40 \mathrm{~cm}\right.$ Agilis EPI ${ }^{\mathrm{TM}}$, Abbott Laboratories, Chicago, IL; or $45 \mathrm{~cm}$ SupraCross ${ }^{\circledR}$; Baylis Medical) to engage the fossa ovalis, as validated by ICE imaging, and on the use of dedicated radiofrequency (RF) access wires (SupraCross ${ }^{\circ}$ RF Wire; Baylis Medical) for puncture.

Because the body of the steerable sheath is significantly stiffer than standard nonsteerable sheaths, it is particularly difficult to maintain the curvature of a manually bent Brockenbrough needle once advanced within the sheath. In fact, during our early experience, we simulated advancement of a manually bent Brockenbrough needle within a deflectable sheath

This article is protected by copyright. All rights reserved. 
outside the body, and we were unable to maintain the preformed curvature of the needle within the sheath. This would have resulted in dislodgement of the sheath apparatus away from the transseptal site selected for puncture.

The choice of the steerable sheath is dependent on the individual patient anatomy. The SupraCross $^{\circledR}$ sheath is a unidirectional deflectable sheath with a larger curve, a more flexible dilator, and a smoother transition between dilator and sheath compared to the Agilis $\mathrm{EPI}^{\mathrm{TM}}$ sheath; these features enhance the steerability of the sheath with the dilator in place, facilitate the engagement and tenting of the fossa ovalis from a superior approach, and improve tracking of the sheath over the dilator into the LA. Once transseptal access is obtained, catheter manipulation for mapping and ablation can be easier with the Agilis $\mathrm{EPI}^{\mathrm{TM}}$ sheath, due to its tighter deflection curve and bidirectional steerability.

Figures $\mathbf{2}$ and $\mathbf{4}$ provide a step-by-step illustration of the approach used for transseptal catheterization from a superior access with sequential fluoroscopic images. First, the steerable sheath is advanced over a long J-tipped wire to the floor of the RA, close to the ostium of the coronary sinus (Figure 4A). At this point, the wire is removed and the dilator is pulled back inside the sheath, exposing only its distal portion (Figure 4B). This maneuver is important for two reasons: firstly, the sheath's steerability is enhanced if the dilator is not fully exposed and secondly, this allows the dilator to track over the RF wire once access has been obtained while maintaining the sheath in contact with the interatrial septum. This latter aspect underpins a key technical difference compared to a standard transseptal access from an inferior venous approach. For instance, once the fossa ovalis has been properly engaged from an inferior venous approach, advancement of the entire sheath/dilator apparatus forward will result in a right-to-left movement of the transseptal apparatus, which will facilitate passage into the LA. When the fossa ovalis is engaged from a superior access with the sheath deflected with a more perpendicular angle (often close to 90 degrees, Figure 4B), any forward advancement of the sheath/dilator apparatus will result in a superior-to-inferior (rather than right-to-left) movement along the fossa ovalis, with subsequent loss of position. Therefore, once the fossa ovalis has been punctured, a right-to-left movement of the dilator may be produced only by keeping the sheath deflected and in

This article is protected by copyright. All rights reserved. 
contact with the fossa ovalis and gently re-exposing the dilator by pushing it forward to track over the wire into the LA.

The puncture is performed with a specialized pigtail RF wire (SupraCross ${ }^{\circledR}$ RF Wire; Baylis Medical), which is an insulated extra-stiff 0.035 -inch wire with a flexible atraumatic distal spiral tip. The distal end of the wire contains exposed metal which allows for delivery of a short burst of RF energy (up to 15 Watts for up to 2 seconds) to enable puncture. The use of the SupraCross ${ }^{\circledR}$ RF wire has greatly simplified the puncture technique as it has eliminated the need for wire exchanges following the access to allow crossing of the dilator/sheath apparatus into the LA and tracking within the chamber. Indeed, for our initial cases (i.e., prior to commercial availability of the SupraCross ${ }^{\circledR}$ RF wire), we performed transseptal puncture with a straight endovascular RF wire (PowerWire ${ }^{\mathrm{TM}}$, Baylis Medical) followed by exchange with a stiff pigtail wire (ProTrack $^{\mathrm{TM}}$, Baylis Medical, or TorayGuide ${ }^{\mathrm{TM}}$ wire, Toray Medical) to permit advancement of the sheath over the dilator. ${ }^{14}$ Once the sheath/dilator is in contact with the fossa ovalis, the tip of the SupraCross ${ }^{\circledR}$ wire is exposed outside of the dilator and a short pulse of RF energy is delivered to puncture the septum (Figure 4C). Direct visualization with ICE of fine echogenic "bubbles" within the LA generated by the RF application confirms LA entry (Video 2).

The wire is then advanced into the LA under fluoroscopic and ICE guidance until the full spiral end is deployed free (Figure 4D-E and Videos 3 and 4). At this point, the dilator is advanced within the LA over the wire using the aforementioned technique, and the sheath is subsequently advanced over the dilator to complete LA access (Figure 4F). If the operator encounters difficulty with tracking the sheath over the dilator, it is advisable to redirect the wire anteriorly toward the mitral annulus to obtain a more co-axial angle of access to the LA. Occasionally, we have advanced the SupraCross ${ }^{\circledR}$ wire into the left ventricle to achieve transseptal access from a superior approach; as mentioned earlier, the atraumatic curled distal end allows for advancement of the wire against the LA or ventricular wall without concern for risk of perforation.

Another possible approach is the use of a SafeSept ${ }^{\mathrm{TM}}$ transseptal guidewire (Pressure Products, San Pedro, CA) which can be introduced through a standard Brockenbrough

This article is protected by copyright. All rights reserved. 
transseptal needle while tenting the fossa, the wire assumes a J shape after it pierces the fossa thus allowing for advancement within the LA with minimal risk for perforation. We have never used the SafeSept ${ }^{\mathrm{TM}}$ wire for superior access, but we would anticipate challenges in the advancement of the transseptal dilator and sheath within the left atrium over the SafeSept ${ }^{\mathrm{TM}}$ wire, as the body of the wire may not be stiff enough and provide adequate support.

\section{How to Perform Atrial Fibrillation Ablation from a Superior Approach}

Although a dual transseptal access to the LA using the technique described above is feasible, for simplicity, we have performed most of our superior approach AF ablation cases with a single transseptal access and exchanging the multipolar mapping and ablation catheters through the same transseptal sheath (Figure 5). For mapping, we prefer to use multipolar catheters that can be safely manipulated within close proximity of the mitral annulus (e.g., Pentaray ${ }^{\circledR}$ catheter, Biosense Webster, Figure 5A) or even introduced into the left ventricle if needed. In this regard, if during the procedure, the sheath is inadvertently pulled back into the RA with the mapping catheter still within the LA, re-advancement of the sheath over the catheter into the LA is not difficult if a coaxial angle of access is maintained by deployment of the catheter toward the lateral mitral annulus or within the left ventricle, similar to the situation discussed prior with the SupraCross ${ }^{\circledR}$ wire. With respect to the ablation catheter, we have preferred the use of catheters with contact force-sensing technologies to enhance safety of mapping and ablation within the LA, particularly at the LA roof and superior PVs (see further below). Contact forcesensing further allows validation of stable contact at specific regions of the LA, such as the anterior and inferior segments of the right PVs, as the approach to reach these regions from a superior access may require the use of combined sheath and catheter loops with tight deflections, resulting in minimal to complete lack of tactile feedback to the operator.

It is important to realize that the angle of sheath entry into the LA from a superior access differs to what is typically encountered with a conventional inferior venous approach. In order to minimize the risk of losing transseptal access, the deflectable sheath is usually directed toward the center of the LA and then deflected superiorly to allow for mapping of the PVs and posterior LA. In general, the specific catheter movements necessary to cannulate the left and right PVs and

This article is protected by copyright. All rights reserved. 
to map various segments of the LA are reversed compared to the traditional inferior venous approach. For instance, any forward advancement of the sheath and catheter will produce a superior-to-inferior and posterior-to-anterior catheter movement within the LA and reduce contact with the superior regions of the LA and the posterior wall. On the other hand, whenever the catheter or sheath are pulled back, the operator should pay close attention to the contact with the posterior LA and roof regions, as very high contact forces can be easily attainable with minimal movements. In addition, clockwise rotation of the sheath and catheter will reach the anterior segments of the LA, whereas counterclockwise torque will access the posterior segments.

Mapping and ablation of the left PVs and right superior PV can usually be performed via a direct approach (Figure 6A-C), that is, by simply maintaining the sheath in the center of the LA with superior deflection, as aforementioned, and advancing the catheter to map and ablate different aspects of the PVs. The most challenging region for mapping and ablation is the septal segment of the right PVs. If the LA access is properly performed at the superior and anterior aspect of the fossa ovalis, a direct approach with the sheath slightly pulled back close to the access site while maintaining counterclockwise torque is typically sufficient to perform ablation of the anterior right PV roof and septum up to the anterior carina region. Ablation of the anterior segments of the right inferior PV below the region of the carina with a direct approach is generally not possible and is also associated with a significant risk of losing transseptal access. Therefore, we prefer to approach ablation of the anterior segments of the right inferior PV by creating a large catheter loop within the body of the LA and then deflecting the catheter anteriorly to achieve contact with the septum. The loop is formed by directing the sheath toward the infero-lateral mitral annulus and maximally deflecting it to point backwards towards the septum. The catheter is then advanced and deflected anteriorly (Figure 7A). In patients with larger LA sizes, reaching the most inferior portions of the septum may be challenging even after creating a loop with the deflectable sheath; in these cases, forming a secondary loop with the catheter (Figure 7B) is usually adequate to achieve stable contact with the inferior septum and complete the ablation.

This article is protected by copyright. All rights reserved. 


\section{Conclusions}

We describe our technique to perform LA transseptal catheterization and AF ablation via a superior venous access under ICE guidance, using steerable sheaths and dedicated RF wires. This approach has permitted catheter ablation of AF in patients with IVC-I in whom a standard procedure from a transfemoral venous approach is not feasible.

\section{References}

[1] Calkins H, Hindricks G, Cappato R, Kim YH, Saad EB, Aguinaga L, Akar JG, Badhwar V, Brugada J, Camm J, Chen PS, Chen SA, Chung MK, Nielsen JC, Curtis AB, Davies DW, Day JD, d'Avila A, de Groot N, Di Biase L, Duytschaever M, Edgerton JR, Ellenbogen KA, Ellinor PT, Ernst S, Fenelon G, Gerstenfeld EP, Haines DE, Haissaguerre M, Helm RH, Hylek E, Jackman WM, Jalife J, Kalman JM, Kautzner J, Kottkamp H, Kuck KH, Kumagai K, Lee R, Lewalter T, Lindsay BD, Macle L, Mansour M, Marchlinski FE, Michaud GF, Nakagawa H, Natale A, Nattel S, Okumura K, Packer D, Pokushalov E, Reynolds MR, Sanders P, Scanavacca M, Schilling R, Tondo C, Tsao HM, Verma A, Wilber DJ, Yamane T: 2017 HRS/EHRA/ECAS/APHRS/SOLAECE expert consensus statement on catheter and surgical ablation of atrial fibrillation. Heart rhythm : the official journal of the Heart Rhythm Society 2017; 14:e275-e444.

[2] Link MS, Exner DV, Anderson M, Ackerman M, Al-Ahmad A, Knight BP, Markowitz SM, Kaufman ES, Haines D, Asirvatham SJ, Callans DJ, Mounsey JP, Bogun F, Narayan SM, Krahn AD, Mittal S, Singh J, Fisher JD, Chugh SS: HRS policy statement: clinical cardiac electrophysiology fellowship curriculum: update 2011. Heart rhythm : the official journal of the Heart Rhythm Society 2011; 8:1340-1356.

[3] Ross J, Jr., Braunwald E, Morrow AG: Trans-septal left atrial puncture: a new method for the measurement of left atrial pressure in man. Am J Cardiol 1959; 3:653655.

[4] Ross J, Jr., Braunwald E, Morrow AG: Transseptal left heart catheterization: a new diagnostic method. Progress in cardiovascular diseases 1960; 2:315-318.

[5] Braunwald E: Cooperative study on cardiac catheterization. Transseptal left heart catheterization. Circulation 1968; 37:III74-79.

[6] Brockenbrough EC, Braunwald E, Ross J, Jr.: Transseptal left heart catheterization. A review of 450 studies and description of an improved technic. Circulation 1962; 25:15-21.

[7] Mullins CE: Transseptal left heart catheterization: experience with a new technique in 520 pediatric and adult patients. Pediatric cardiology 1983; 4:239-245.

This article is protected by copyright. All rights reserved. 
[8] Winkle RA, Mead RH, Engel G, Patrawala RA: The use of a radiofrequency needle improves the safety and efficacy of transseptal puncture for atrial fibrillation ablation. Heart rhythm : the official journal of the Heart Rhythm Society 2011; 8:1411-1415.

[9] Crystal MA, Mirza MA, Benson LN: A radiofrequency transseptal needle: initial animal studies. Catheterization and cardiovascular interventions : official journal of the Society for Cardiac Angiography \& Interventions 2010; 76:769-773.

[10] Tucker KJ, Curtis AB, Murphy J, Conti JB, Kazakis DJ, Geiser EA, Conti CR:

Transesophageal echocardiographic guidance of transseptal left heart catheterization during radiofrequency ablation of left-sided accessory pathways in humans. Pacing and clinical electrophysiology : PACE 1996; 19:272-281.

[11] Epstein LM, Smith T, TenHoff H: Nonfluoroscopic transseptal catheterization: safety and efficacy of intracardiac echocardiographic guidance. Journal of cardiovascular electrophysiology 1998; 9:625-630.

[12] Kato H, Kubota S, Yamada Y, Kumamoto T, Takasawa Y, Takahashi N, Yamamoto $\mathrm{M}$ : Circumferential pulmonary vein ablation of atrial fibrillation via superior vena cava approach in a patient with interruption of the inferior vena cava. Europace : European pacing, arrhythmias, and cardiac electrophysiology : journal of the working groups on cardiac pacing, arrhythmias, and cardiac cellular electrophysiology of the European Society of Cardiology 2010; 12:746-748.

[13] Lim HE, Pak HN, Tse HF, Lau CP, Hwang C, Kim YH: Catheter ablation of atrial fibrillation via superior approach in patients with interruption of the inferior vena cava. Heart rhythm : the official journal of the Heart Rhythm Society 2009; 6:174-179.

[14] Betensky BP, Santangeli P: Radiofrequency wire-facilitated transseptal access using a superior approach for atrial fibrillation ablation in a patient with inferior vena cava obstruction. HeartRhythm Case Rep 2016; 2:265-267.

This article is protected by copyright. All rights reserved. 


\section{FIGURE LEGENDS}

Figure 1. Example of procedure room setup during a left axillary vein access (Panels $A$ and $B$ ) and right internal jugular vein access (Panels $C$ and $D$ ) AF ablation. Panel $A$ : Left axillary vein access is used for the transseptal sheath and ICE catheter. Panel $B$ : To improve ergonomics a second table is positioned at the side of the patient's table to facilitate manipulation of the deflectable sheath and ablation catheter and maintain a coaxial angle of access to the axillary vein. Panel C: Right internal jugular vein access is used for the transseptal sheath and a separate right axillary vein access is used for the ICE catheter and the diagnostic coronary sinus catheter. Panel D: Manipulation of the deflectable sheath and mapping/ablation catheter from a right internal jugular vein approach is less favorable compared to a left axillary vein approach. The operator stands at the head of the table while holding the deflectable sheath and catheter in place to maintain a coaxial angle of approach to the right internal jugular vein.

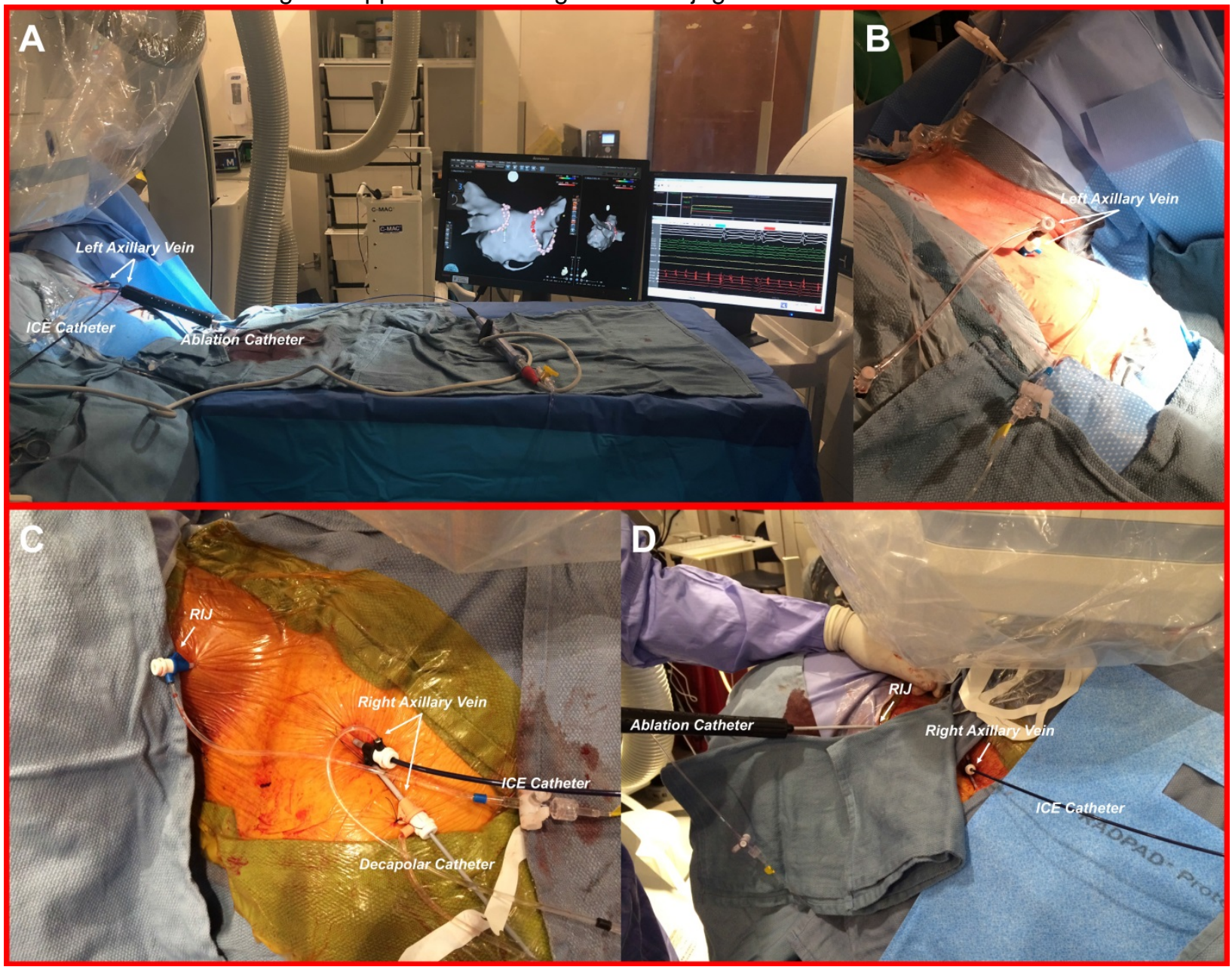

This article is protected by copyright. All rights reserved. 
Figure 2. Schematic illustrating the technique for left atrial access from a superior approach (axillary vein). The deflectable sheath is advanced to the right atrium and deflected to engage the fossa ovalis (Panel $A)$. Once proper tenting of the fossa ovalis has been verified with intracardiac echo, the Supracross $\AA$ wire is exposed and RF is applied to obtain access. The wire is advanced to the LA to deploy the full spiral (Panel $B)$ and allow for the dilator and sheath to cross to the LA (Panel C). The specific anatomical relationships relevant to the transseptal access are similar to what typically encountered with a standard left atrial transseptal catheterization from a femoral venous approach.
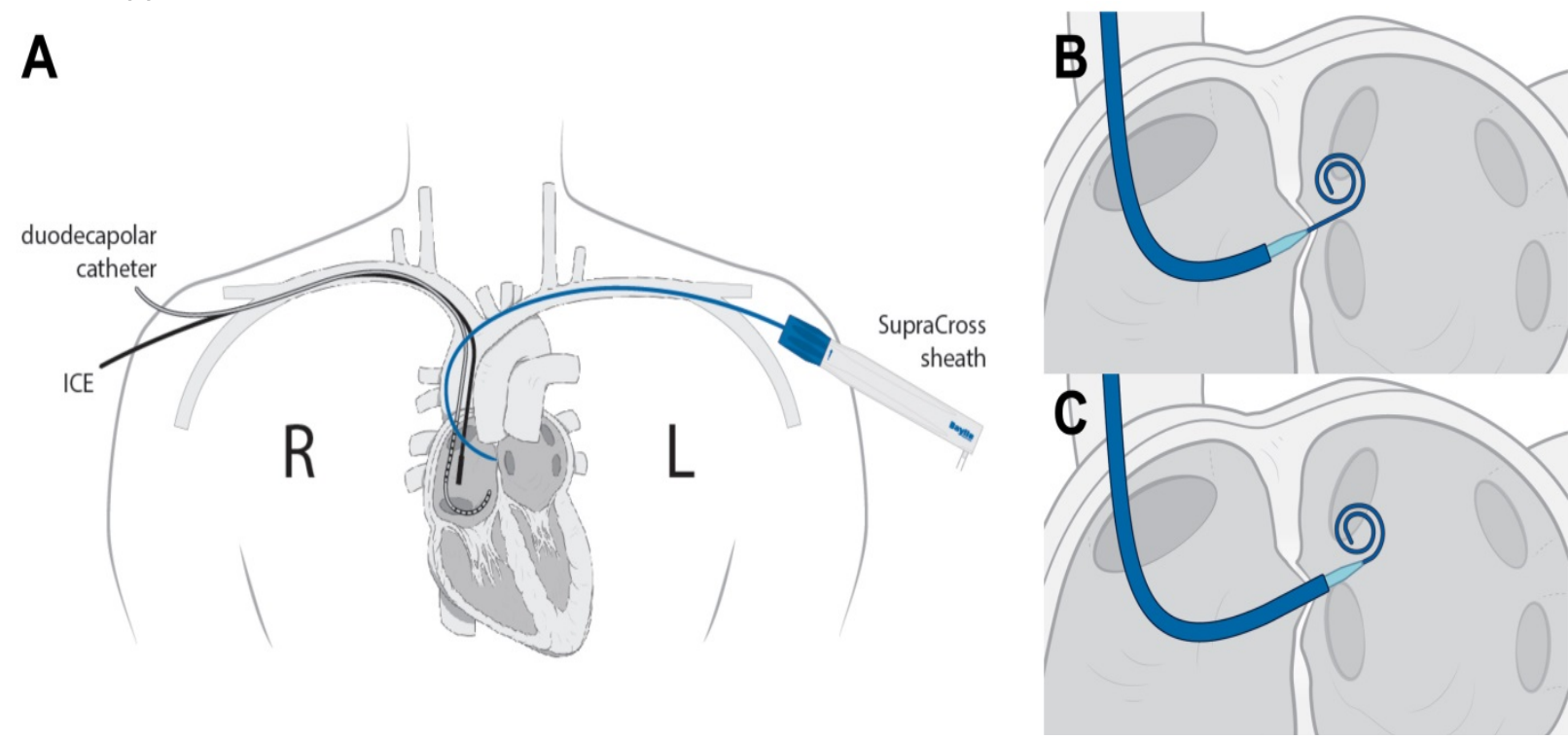

Figure 3. ICE image showing the sheath and dilator positioned at the superior and anterior aspect of the fossa ovalis to obtain LA transseptal access from a superior approach. MV = mitral valve; $L A A=$ left atrial appendage; $R A=$ right atrium; $L A=$ left atrium; $L V=$ left ventricle.

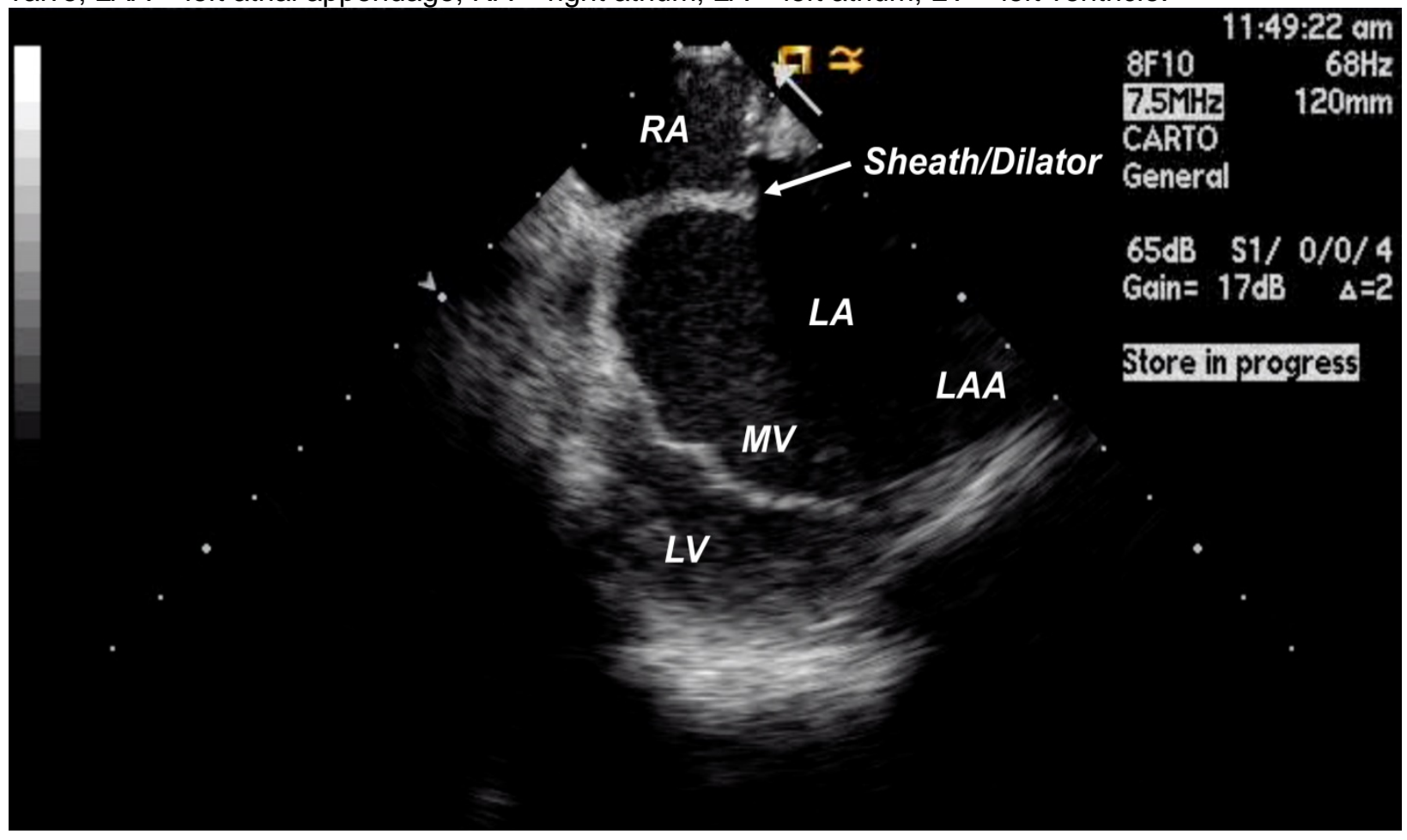

This article is protected by copyright. All rights reserved. 
Figure 4. Step-by-step illustration of the approach used for transseptal catheterization from a superior access. Panel $A$ : the deflectable sheath is advanced to the floor of the RA over a long guidewire with the dilator fully exposed. Panel $B$ : the wire is removed and the dilator is pulled back within the sheath. The sheath is then deflected to an almost 90-degree angle while applying counterclockwise rotation to facilitate engagement of the superior and anterior portion of the fossa ovalis. Panel C: once the fossa ovalis has been engaged, the tip of Supracross ${ }^{\circ}$ RF wire is exposed to puncture the septum. Panel $D$ : radiofrequency is applied to the tip of the Supracross $®$ wire and the wire is advanced within the LA until the full wire spiral is deployed. Panels E-F: to facilitate advancement of the dilator and sheath in the LA, the wire is advanced to the mitral annulus to obtain a more coaxial angle of access to the LA.

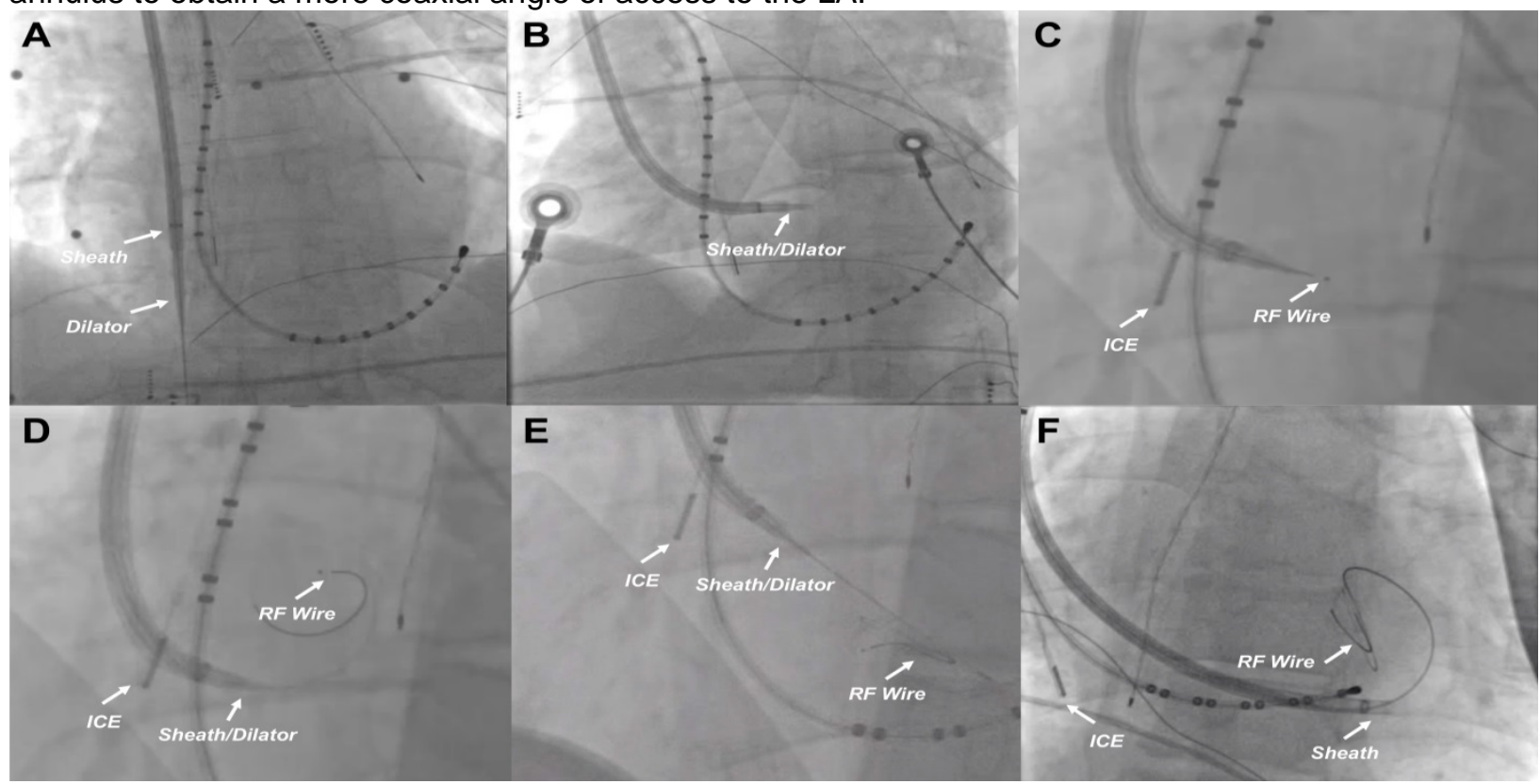

Figure 5. Single transseptal access with sequential LA mapping (Panel $A)$ and ablation (Panel $B)$.

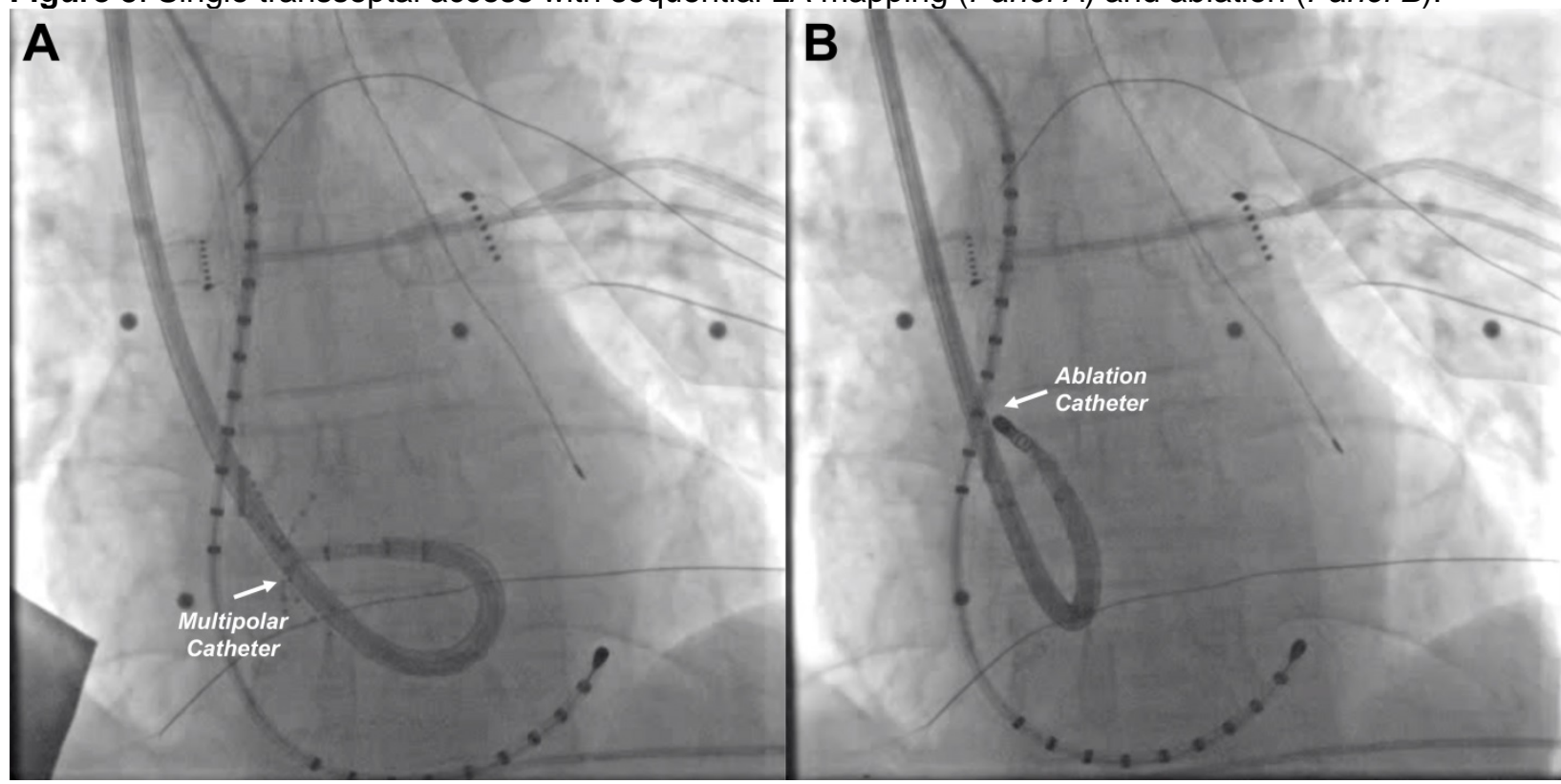

This article is protected by copyright. All rights reserved. 
Figure 6. Example of approach used to map and ablate the left pulmonary veins (Panels $A$ and $B$ ) and the right superior pulmonary vein $(P a n e l C)$. This can be usually accomplished via a direct approach by maintaining the sheath in the body of the LA and advancing the catheter to map and ablate different aspects of the PVs.
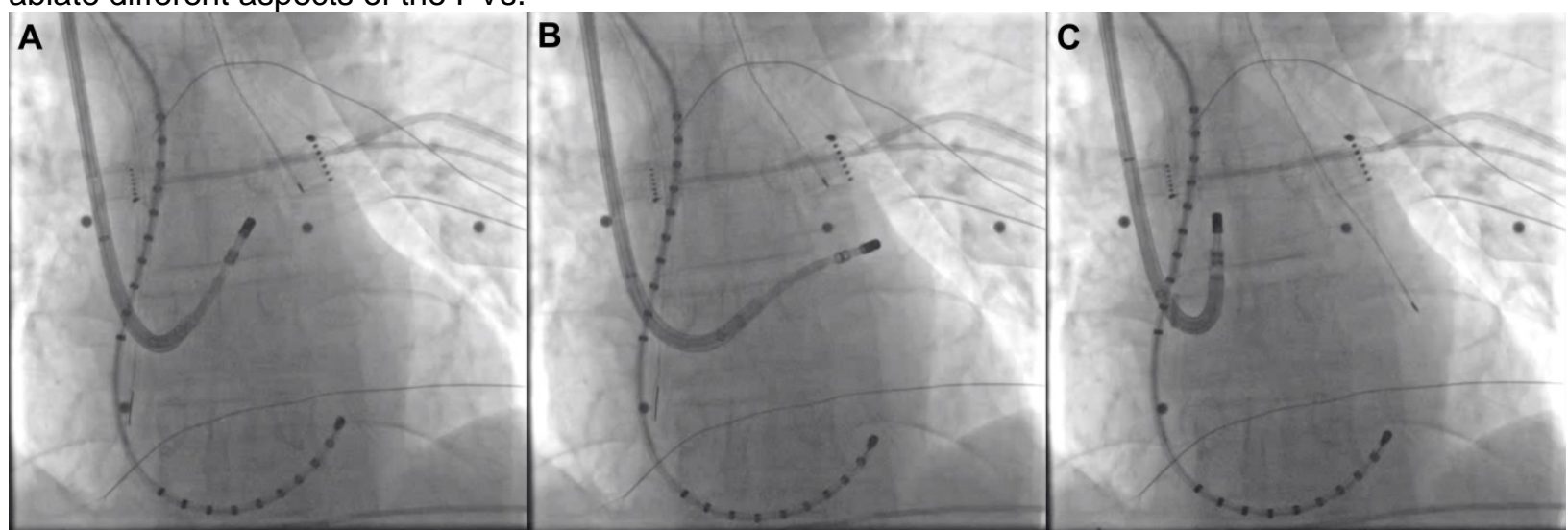

Figure 7. Example of approach to map and ablate the anterior segments of the right inferior PV. Typically, this is best accomplished by creating a large loop with the sheath (Panel $A$ ) and/or catheter (Panel B).

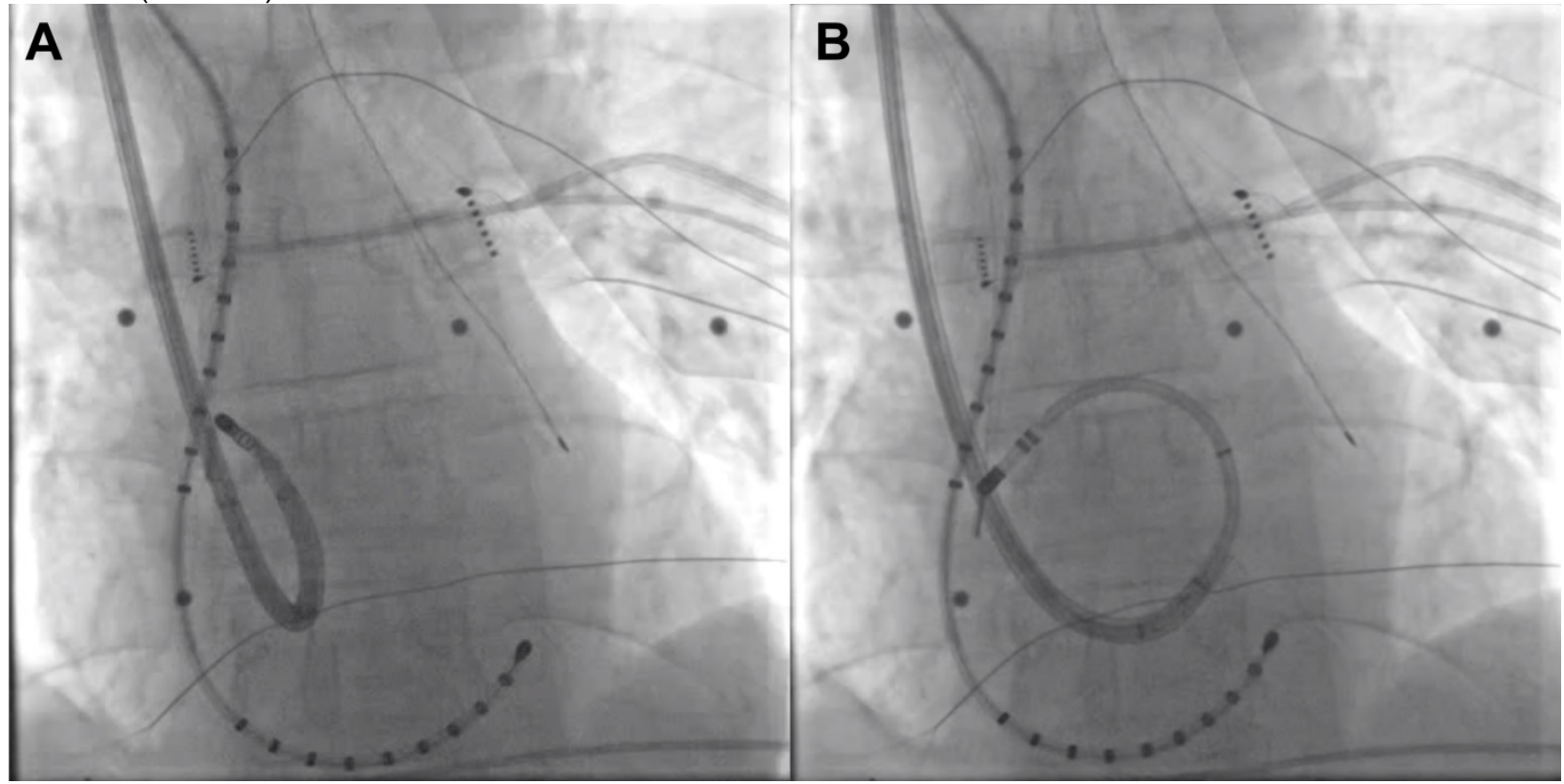

This article is protected by copyright. All rights reserved. 